\title{
Improving the Classifier Performance in Motor Imagery Task Classification: What are the steps in the classification process that we should worry about?
}

\author{
Miriam Seoane Santos ${ }^{1}$, Pedro Henriques Abreu ${ }^{1}$, Germán Rodríguez-Bermúdez² ${ }^{2}$ Pedro J. \\ García-Laencina $^{2}$ \\ ${ }^{1}$ Centre for Informatics and Systems, University of Coimbra, \\ Pólo II, Pinhal de Marrocos, Coimbra, 3030-290, Portugal \\ E-mail:miriams@student.dei.uc.pt,pha@dei.uc.pt \\ ${ }^{2}$ University Centre of Defence at the Spanish Air Force Academy, UPCT-MDE \\ Calle Coronel Lopez Pe $\widetilde{n}$, s/n, Santiago de la Ribera, Murcia, 30720, Spain
}

E-mail: german.rodriguez@cud.upct.es

Received 28 February 2018

Accepted 11 July 2018

\begin{abstract}
Brain-Computer Interface systems based on motor imagery are able to identify an individual's intent to initiate control through the classification of encephalography patterns. Correctly classifying such patterns is instrumental and strongly depends in a robust machine learning block that is able to properly process the features extracted from a subject's encephalograms. The main objective of this work is to provide an overall view on machine learning stages, aiming to answer the following question: "What are the steps in the classification process that we should worry about?". The obtained results suggest that future research in the field should focus on two main aspects: exploring techniques for dimensionality reduction, in particular, supervised linear approaches, and evaluating adequate validation schemes to allow a more precise interpretation of results.
\end{abstract}

Keywords: Brain Computer Interface Systems; Motor Imagery Tasks; Pattern Recognition; Machine Learning.

\section{Introduction}

A Brain-Computer Interface (BCI) is a communication system that uses electroencephalography (EEG) signals to extract, decode and translate intentions into commands. When they first were developed, the may purpose of BCI systems was to enable the interaction of severe motor disabled people with devices such as computers, speech synthesisers or neural prostheses, which gave the chance for tetraplegic people, individuals with amyotrophic lateral sclerosis, brain stem stroke or spinal cord injury to achieve a higher independence status, improving their quality of life. Today, there are many applications for $\mathrm{BCI}$ to control external devices such as robots, com- munication systems, games, virtual reality or rehabilitative applications 112 . BCIs are also applied to user-state monitoring or for the evaluation of neuromarketing applications, in training or education programs, for cognitive improvement or in safety and security applications ${ }^{2}$. In summary, these systems detect specific patterns of a person's EEG that relate to their intentions to initiate control and translate them into meaningful control commands. The detection of these patterns is crucial and depends on a strong component of signal processing and classification.

Typically, a BCI system (Fig. 1) can be seen as an artificial intelligence system 3 that follows five consecutive stages: a) EEG signal generation, b) 
EEG signal acquisition and preprocessing, c) Feature Extraction, d) Classification and finally e) Applications. Although all parts are important in BCI systems, it is obvious that a good design of the machine learning block is necessary to obtain a good performance. Into the bargain, the quality of predictions depends on both the extracted features and the classification algorithm employed. Several different methods can be applied to extract features from channels located at specific brain areas, such as Spectral Parameters, Parametric Modelling, Signal Envelope, Signal Complexity or some combination of different feature extraction methods 1/3445. Thus, classification algorithms have to manage large feature vectors even when the information provided by some features is not relevant and it increases the complexity of the classifier design. Therefore, as it is shown in the literature, researchers have applied Feature Selection (FS) techniques such us Genetic Algorithms or Feature Transformation (FT) approaches such as Principal Component Analysis (PCA), Independent Component Analysis (ICA), or Common Spatial Patterns (CSP) $\frac{1,6778}{1 n}$ in order to get the most discriminative features. Also, linear classifiers, neural networks, nonlinear Bayesian classifiers, nearest neighbour and combinations of them $1 / 3$ have been tested, under different sampling strategies, to improve the classification accuracy results. However, there is not a conclusion about which is the best way to design the machine learning stage since each author decides which steps and techniques to apply in each work.

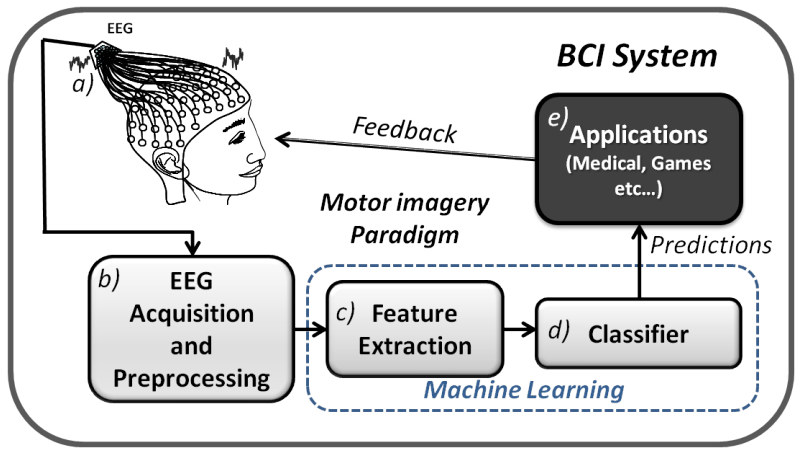

Fig. 1. General scheme of a BCI system.

There are different control signal types in BCI: visual evoked potentials, slow cortical potentials,
P300 evoked potentials, and sensorimotor rhythms 1]. One of the most widely studied EEG-based BCI paradigms is the motor-imagery-based BCI 9 . Motor Imagery (MI) is defined as an imagining of kinesthetics movements, and it is well known to modulate sensorimotor rhythm around the motor cortex 10 .

In this work, EEG signals acquired under MI paradigms have been processed. These are BCI signals from BCI competitions II and III, which represent standard datasets to test signal processing methods and algorithms. The overall goal of this work is to study several pattern recognition approaches able to select/extract relevant features from BCI datasets and identify the patterns that correspond to an individual's intent of initiating control (a binary classification problem). Thus, stages (c) and (d) will be analysed in a deeper way in order to answer this question: "What are the steps in the classification process that we should worry about?".

The contribution of this research is therefore two-fold: for researchers familiarised with the field, it provides a comprehensive comparison of approaches included in several stages of the machine learning block comprised in BCI systems; for researchers new to the field, it provides an extensive description and illustration of a complete experimental setup in BCI domains, from the data collection stage to the final classification task, including a thorough explanation of all the techniques frequently used along the process.

The manuscript is organised as follows. Section 2 covers the experimental setup and a brief background on the algorithms used in this work. Section 3 presents and discusses the obtained results. Finally, in Section 4 some conclusions and future directions are discussed.

\section{Experimental Setup}

In this work, in order to reflect the most usual steps applied from a machine learning perspective in BCI systems, the Machine Learning block has been expanded in six different stages: 1) Feature Computation, 2) Feature Preprocessing, 3) Feature Selection, 4) Feature Transformation/Projection, 5) Classification Task and finally 6) Performance Evalua- 
tion (Fig. 2). Stages 1 and 2 consider the implementation of well-established approaches to BCI data, in what concerns feature extraction (Power Spectrum Density, Hjorth Parameters and Adaptive and Autoregressive Parameters) and feature preprocessing (Z-score normalisation). This work mainly investigates concurring approaches from stages 3 to 6 , where several different techniques are compared: in stage 3, five techniques have been tested for feature selection (Area Under the ROC Curve, Fisher Score, Information Gain, Point Biserial Correlation and Kruskal-Wallis test); in stage 4, three methods were studied for feature transformation (Principal Component Analysis, Linear Discriminant Analysis and Isomap); in stage 5, four different machine learning classifiers were considered (Fisher Linear Discriminant, Naïve Bayes, Support Vector Machines and Decision Trees) and finally, in stage 6, three validation methods were applied (Holdout, k-fold crossvalidation and Leave-One-Out). The main aspects of each stage are discussed below.

\subsection{Data Description and Feature Computation}

This work focuses on the labeled data of four BCI datasets from four different subjects (Table 1). The data comes from "BCI Competitions" that are widely known in BCI research. The goal of these competitions is to validate signal processing and classification methods for Brain Computer Interfaces (BCIs) 11 .

All the signals used in this work were collected with a g.tec amplifier working at $128 \mathrm{~Hz}$, under a MI paradigm: in particular, the movement of right $(\mathrm{RH})$ or left hand (LH). In all experiments, three bipolar channels located over $\mathrm{C} 3, \mathrm{Cz}$ and $\mathrm{C} 4$ were used, although only signals from $\mathrm{C} 3$ and $\mathrm{C} 4$ filtered between 0.5 and $30 \mathrm{~Hz}$ were chosen to compute the feedback signals. The data of Subject 1 has been extracted from BCI Competition II (set III). In this experiment, the subject was relaxed in a chair looking at a screen: each trial started with a black screen and two seconds later, an acoustic signal marked the beginning of the trial (Fig. 3). Then, a cross appeared during one second and an arrow (left or right) was displayed as a cue. At this moment, the user imagined the movement of the corresponding hand to move a bar into the direction of the cue. The bar reflects the real time output of the classifier along the feedback period and it was computed based on adaptive autoregressive parameters (AAR) and a discriminant analysis 11 .

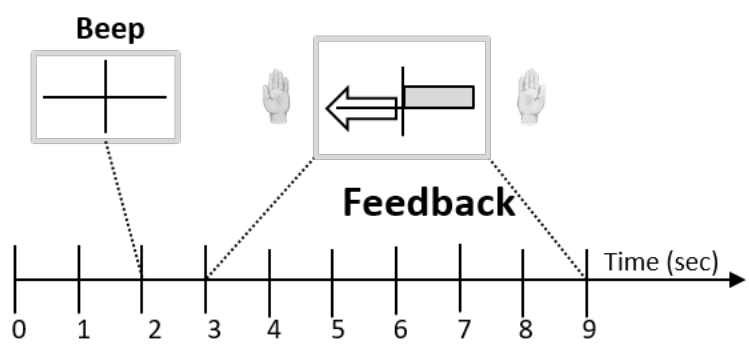

Fig. 3. Timing of bar feedback: BCI Competition II, set III.

The data from the $2^{\text {nd }}, 3^{\text {rd }}$ and $4^{\text {th }}$ subjects comes from BCI competition III (set IIIb). For this experiment, the timing and the feedback was slightly different. In the beginning of each trial, the screen was black until a cross appeared after two seconds. Then, an acoustic signal attracted the user's attention, a visual cue appeared and feedback started. Subjects 2 and 3 used basket feedback (Fig. 4) computed with AAR and an adaptive quadratic discriminant analysis. Basket feedback consisted of a ball falling down from the centre top of the screen, and the user should try to move the ball to the left or right depending on its colour (green for left or red for right). Subject 4 used also a bar feedback computed using alpha and beta band power and a linear discriminant analysis. 12 .

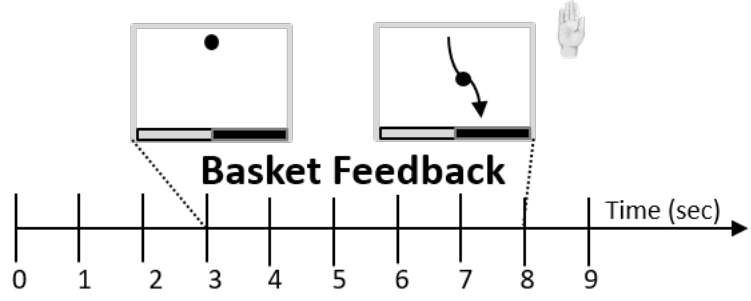

Fig. 4. Timing of basket feedback: BCI Competition III, set IIIb.

In order to take advantage of the complementary information provided by different feature extraction methods, for all subjects three feature Extraction methods have been applied in channels $\mathrm{C} 3$ 


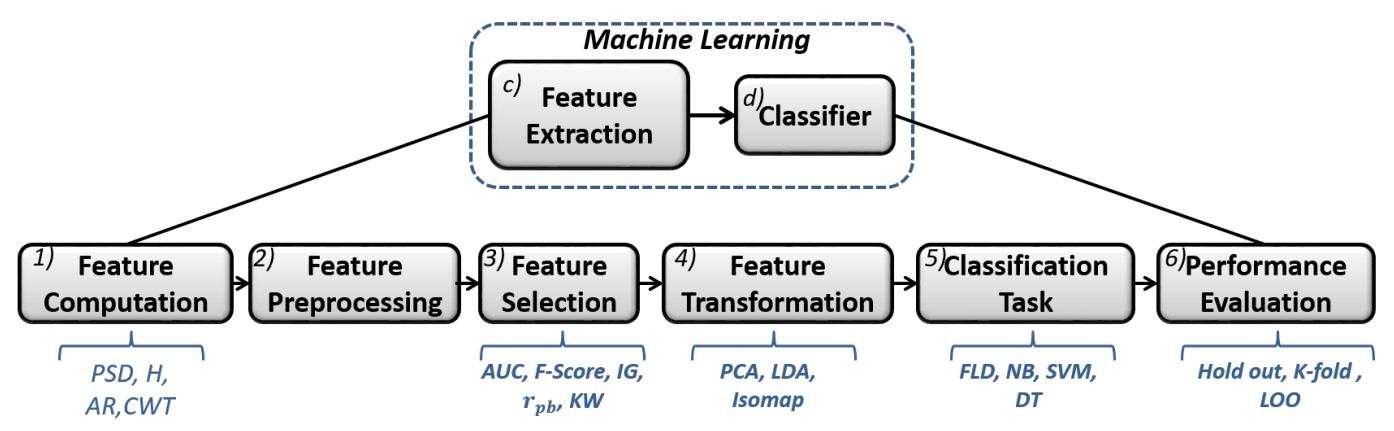

Fig. 2. Proposed experimental setup.

and C4: Power Spectral Density (PSD) in alpha and beta frequency bands, Hjorth parameters (Activity, Mobility and Complexity), Auto Regressive (AR) model coefficients of six order and Continuous Wavelet Transform coefficients (CWT) using a standard "Daubechies D2" wavelet type with six features per channel, as done in previous works. $\frac{8|13| 14}{\text {. }}$ These FE methods have been widely applied in BCI systems and they are very representative of MI tasks 4. Features have been computed in a standard 1 second window in the MI period ${ }^{3}$, and therefore, there is a vector with 34 features per trial. It is important to remark that the analysis of the feature computation is not the objective of this work.

Table 1. Dataset description for all 4 subjects.

\begin{tabular}{ccccc}
\hline Subject & Dataset origin & Trials & Classes & Feedback \\
\hline 1 & BCI comp. II & 140 & 2 & Bar \\
2 & BCI comp. III & 320 & 2 & Basket \\
3 & BCI comp. III & 540 & 2 & Basket \\
4 & BCI comp. III & 540 & 2 & Bar \\
\hline
\end{tabular}

\subsection{Feature Preprocessing}

Data preprocessing is performed to reduce noise and increase the consistency of data. The most common preprocessing steps used in the literature are Normalisation or Standardisation of data. Normalisation (Min-Max transformation) refers to the feature scaling between its minimum and maximum values, while Standardisation (Z-Score transformation) transforms the features so that they have a zero mean and unitary standard deviation. The objective of normalisation/standardisation is to make features with different scales and ranges of measurement comparable, so that none has more influence than the others on classification task. In this work, the Z-Score transformation (equation 1) ${ }^{15}$ was applied, which is a common feature processing algorithm for BCI 14 . In equation 1, $x_{i}$ is each value from a given feature, and $\mu$ and $\sigma$ are the mean and standard deviation of such feature, respectively. Z-score is applied as a standard procedure for data cleaning $16 \mid 17$, since this stage is not the main focus of our analysis.

$$
z_{i}=\frac{x_{i}-\mu}{\sigma}
$$

\subsection{Feature Selection}

After preprocessing, a common procedure is to reduce the dimensionality of the input data in order to ease the classification task. This can be done by assessing the discriminative quality of the input features, called Feature Selection (FS), or by Feature Transformation (FT), where the input data is projected onto a low dimensional space 18/19|20|21. FS techniques keep the original meaning of the input features, thus providing a good interpretation of the low-dimensional space: the selected features will be a set of the most discriminative and relevant features for the MI problem. Besides, once the most discriminative features are determined, only them need to be computed for new subjects. In this paper we have computed five FS techniques: AUC values, F-score, IG, $r_{\mathrm{pb}}$ and $\mathrm{KW}$. These approaches are representative from a machine learning perspective and they have been previously used in BCI systems $7722|23| 24|25| 26$. We now describe the different FS techniques ex- 
plored in this work.

\subsubsection{AUC values}

The Area Under the ROC curve (AUC) is most often used as performance measure for classification. The Receiver Operating Characteristics curve (ROC) is a plot that represents the trade-offs between the classifier's true positive rate (TPR) in the $y y$ axis, and false positive rate (FPR) in the $x x$ axis and thus it informs on the variation of the classifier's sensitivity and specificity for different cut-off values (classification thresholds) 27. Given that both TPR and FPR range from 0 to 1 , the AUC of an ideal classifier is 1 . Therefore, the discriminant power of a classifier is associated to the area under that curve: the higher the area is, the better. However, the AUC can also be used to determine the discriminative power of the existing features: instead of the classifier outputs (scores), the feature values are used to build the ROC curve. Just the same, the bigger the AUC is, the more discriminative the feature is.

\subsubsection{F-score}

The Fisher score (F-score) is one of the most widely used supervised feature selection methods, and measures the overlap between individual feature values of different classes (RH, LH), where higher values indicate more discriminative features 28 . Considering two classes $\mathrm{RH}$ and $\mathrm{LH}$, the Fisher score of a feature $j$ is given by equation 2 , where $\mu_{R H, j}, \mu_{L H, j}$, $\sigma_{R H, j}$ and $\sigma_{L H, j}$ are the means and standard deviations of classes $\mathrm{RH}$ and $\mathrm{LH}$, respective to the $j$-th feature.

$$
\operatorname{F-score}(j)=\frac{\left(\mu_{R H, j}-\mu_{L H, j}\right)^{2}}{\sigma_{R H, j}^{2}+\sigma_{L H, j}^{2}}
$$

\subsubsection{Information Gain}

Information Gain (IG) measures the ability of a given feature to discriminate the input patterns (trials), according to their target $(\mathrm{RH}, \mathrm{LH}) \underset{29}{29}$. It is an entropy-based measure, most often used for selecting the best features at each step in growing a decision tree (e.g. ID3 algorithm). Considering a collec- tion of trials $D$, containing examples of $\mathrm{RH}$ and $\mathrm{LH}$ movements, the necessary information to classify a trial in $D$ is defined as $I(D)$, or $E(D)$ (the entropy of $D$ ), which measures the impurity of the collection $D$. $E(D)$ would be 0 if all trials in $D$ belonged to the same class, and 1 if RH and LH were equally represented. Establishing entropy as a definition of impurity, we can measure the effectiveness of a feature $j$ to discriminate classes $\mathrm{RH}$ and $\mathrm{LH}$, by computing the entropy of $D$ after using feature $j$ to divide $D$ in several partitions, $E(D, j)$. The effectiveness of a feature (its discriminative power) is therefore given by equation 3 . The top discriminative features are determined by ranking their information gains, for all features in the dataset.

$$
\operatorname{Gain}(j)=E(D)-E(D, j)
$$

\subsubsection{Point Biserial Correlation}

The Point Biserial correlation coefficient $\left(r_{p b}\right)$ is the most appropriate correlation index between a continuous (interval or ratio-scaled) feature and a dichotomous (binary) feature ${ }^{30}$. Considering the motor imagery problem in this work, $r_{p b}$ can be used to assess the discriminative power of each feature $j$, by determining its correlation with the class label $(\mathrm{RH}$, $\mathrm{LH}): r_{p b}$ varies from 0 to 1 , where 0 indicates that there is no correlation and 1 indicates an elevated degree of correlation. The point-biserial correlation of each feature can be computed using equation 4 , where $\mu_{R H, j}$ and $\mu_{L H, j}$ are the means of feature $j$ for all RH and LH trials, respectively; $\sigma_{j}$ is the standard deviation of feature $j ; n_{R H}$ and $n_{L H}$ are the number of trials belonging to $\mathrm{RH}$ and $\mathrm{LH}$ classes and $n$ is the total number of trials.

$$
r_{p b}(j)=\frac{\mu_{R H, j}-\mu_{L H, j}}{\sigma_{j}} \sqrt{\frac{n_{R H} n_{L H}}{n^{2}}}
$$

\subsubsection{Kruskal-Wallis Test}

The Kruskal-Wallis (KW) test is a non-parametric test used to compare the distribution of two or more features observed in two or more independent samples. It can be used to test if two or more samples come from the same population or from different 
populations; in other words, if the samples come from populations with the same distribution 31 . The $\mathrm{Qui}^{2}$ values are a result of KW statistics and can be used to rank the features according to their discriminative power: the higher the Qui ${ }^{2}$ value, the more discriminative the feature is.

\subsection{Feature Transformation}

Instead of selecting a subset of the most discriminative features, Feature Transformation (FT) methods are able to "combine" the features and project the data onto a different coordinate system, in such a way that the redundancy between features is minimised, and only relevant information is kept. This ability to "combine" the input features, as a higher level of feature fusion, usually increases the classification accuracy 14, which makes FT methods so attractive. However, the data dimensions in the low dimensional space are the result of a combination of the original dimensions (features), which means that the new dimensions (components) do not have any physical interpretation in the problem under study. In this work, three commonly used FT methods in BCI applications are studied 132: Principal Component Analysis, Linear Discriminant Analysis and Isomap, described herein.

\subsubsection{Principal Component Analysis}

Principal Component Analysis (PCA) is an linear, unsupervised dimensionality reduction method, whose objective is to produce a low-dimensional representation of the original input space 14 . PCA transforms the set of $p$ correlated original features $\left\{x_{1}, x_{2}, . ., x_{p}\right\}$ in a smaller set of uncorrelated, orthogonal variables, referred to as principal components (not to be confused with features), $\left\{z_{1}, z_{2}, . ., z_{p}\right\}$. The main idea of PCA is to find an orthonormal transformation to provide the best set of projections (i.e. eigenvectors) to represent the structure of data, with the minimum possible redundancy, but without significant loss of information. The projections are chosen in order to retain the maximum amount of information, measured in terms of data variance: the first component explains the maximum variance in data, the second the following maximum variance and so forth (the last component will be the one that contributes the less to explain the total variance in data). There are two main criteria to choose the optimal number of principal components to keep 33. Kaiser criterion and Scree test. Kaiser criterion suggests keeping only components with eigenvalues equal or higher than 1, while the Scree test is based on a plot of the explained variance for each component: it suggests discarding the components starting where the plot levels off. Both criteria are standard approaches for BCI domains $34[35$ and were therefore tested in this work.

\subsubsection{Linear Discriminant Analysis}

Linear Discriminant Analysis (LDA), also called FDA (Fisher Discriminant Analysis) or FLD (Fisher Linear Discriminant) is also a linear transformation technique; yet, while PCA is an unsupervised method, more oriented to feature discrimination and visualisation, LDA is a supervised method that considers class-membership information for data reduction, thus more helpful in data classification ${ }^{14}$. LDA is based on the concept of searching for a linear combination of features that allows the maximisation of between-class variance, while minimising the within-class variance. It is important to mention that, while PCA produces a $n \times p$ matrix ( $n$ being the number of existing patterns and $p$ the number of components), LDA produces a $n \times 1$ vector, where the input space is projected down to one dimension. Although LDA/FDA is a popular method for dimensionality reduction, it can also be used for classification 36. In the context of BCI, this would imply choosing a threshold $\omega_{0}$ such that if the onedimensional projection of a trial $x_{i}$, here referred as $z_{i}$, is higher or equal to $\omega_{0}$, then $x_{i}$ belongs to class $\mathrm{RH}$, and to LH otherwise $\left(z_{i}<\omega_{0}\right)$. Note that, in this work, FLD is not coupled with LDA in the experiments because they give equivalent solutions.

\subsubsection{Isomap}

Given that PCA and LDA are linear transformations, they may not appropriately reflect nonlinear properties of the data, which constitutes their main disadvantage. Unlike these classical techniques, Isomap 
is able to discover nonlinear properties underlying the data 37. Isomap is based on three main steps: in the first step, this algorithm takes the input distances $d_{x}(i, j)$ between all pairs $i, j$ from $N$ data points in the high-dimensional input space $X$, measured either in the standard euclidean metric or in some domain-specific metric. Based on the distances $d_{x}(i, j)$, Isomap determines which points are neighbours on the manifold $M$, using one of two methods: (i) connecting all points within a fixed radius $\varepsilon$ or (ii) connecting all points to all of its $k$ nearest neighbours. Then, these relations are represented as a weighted graph $G$. In the second step, Isomap computes the geodesic distances $d_{M}(i, j)$ between all pairs of points in the manifold $M$ by determining their shortest path distances in the graph G. Finally, Isomap applies the classical MultiDimensional Scaling (MDS) to build a matrix of graph distances, constructing a final embedding of the data in a $d$-dimensional Euclidean space $Y$ that best preserves the estimated intrinsic geometry of data. Similarly to PCA, the intrinsic dimensionality of the data can be estimated by looking for the "elbow" at which the plot of the residual variance of Isomap's dimensions ceases to decrease significantly with added dimensions. To conduct the experiments in this work, the euclidean distance was used as the input-space distance and the $k$ parameter was chosen to build the graph. The choice of $k$ was set to find the smallest value that still leads to an overall connected graph for each dataset.

\subsection{Classification Task}

In this stage, the datasets constructed in the previous phases (FS and FT) are tested with four different classifiers previously used in BCI systems in order to compare results: LDA (or Fisher Linear Discriminant, FLD), Naïve Bayes, Support Vector Machines and Decision Trees 138/39. LDA/FLD was already described in the previous section, therefore, the following subsections refer to the remaining classifiers.

\subsubsection{Nä̈ve Bayes}

Naive Bayes (NB) classifier outputs its predictions based on the posterior probabilities of each existing class, $P\left(\omega_{i} \mid \mathbf{x}\right)$ : the probability that pattern $\mathbf{x}$ belongs to class $\omega_{i}$ 40. In a motor imagery task, two posterior probabilities are considered: the probability that a trial $\mathbf{x}$ represents a $\mathrm{RH}$ movement $\left(\omega_{i}=\right.$ $R H)$, or a LH movement $\left(\omega_{i}=L H\right)$. NB classifier then calculates these posterior probabilities using equation 5, where $P\left(\omega_{i}\right)$ is the prior probability of class $\omega_{i}$, i.e., the probability of occurrence of each class (e.g., proportion of trials that belong to classes $\mathrm{RH}$ or LH); $p\left(\mathbf{x} \mid \omega_{i}\right)$ is called the likelihood of $\mathbf{x}$, which is determined for all feature values in $\mathbf{x}$, given that $\mathbf{x}$ is a $d$-dimensional pattern $\mathbf{x}=\left[x_{1}, x_{2}, \ldots, x_{d}\right]$ : the likelihood of $\mathbf{x}$ is computed through a multiplication of all these individual likelihoods. Finally, $p(\mathbf{x})$ is the total probability of $\mathbf{x}$, and may be determined using equation 6 , although it can be ignored because it is the same for both classes, due to the sum over all classes.

$$
\begin{gathered}
P\left(\omega_{i} \mid \mathbf{x}\right)=\frac{p\left(\mathbf{x} \mid \omega_{i}\right) P\left(\omega_{i}\right)}{p(\mathbf{x})} \\
p(\mathbf{x})=\sum_{i=1}^{c} p\left(\mathbf{x} \mid \omega_{i}\right) P\left(\omega_{i}\right)
\end{gathered}
$$

Given a trial $\mathbf{x}$, NB proceeds to the computation of its posterior probabilities, $P(R H \mid \mathbf{x})$ and $P(L H \mid \mathbf{x})$, and decides on the output based on the maximum posterior probability, that is, if $P(R H \mid \mathbf{x})>P(L H \mid \mathbf{x})$, then the trial belongs to $\mathrm{RH}$, and to $\mathrm{LH}$ otherwise. If the posterior probabilities are equal, then the choice is arbitrary.

\subsubsection{Support Vector Machines}

The concept behind Support Vector Machines (SVM) is to find a discriminant function that maximises the margin of separation between the existing classes (optimal hyperplane), using the information provided by the boundary patterns (called the support vectors) 41 . Considering a motor imagery scenario, where RH and LH classes are linearly separable, the equation of a discriminant hyperplane (decision boundary) would be given by equation 7 , where $g\left(x_{i}\right)$ is the classification label, and $w$ and $b$ are the parameters of the hyperplane (weights and bias that define the plane). 

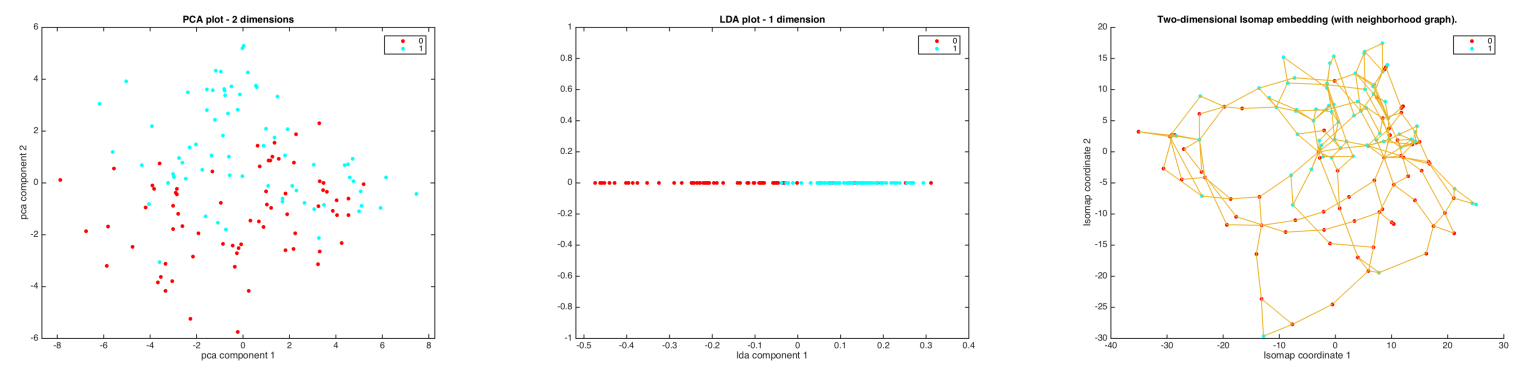

Fig. 5. Feature Transformation techniques for subject 1: PCA, LDA and Isomap embedding. PCA shows a two-dimensional scatter plot considering the two most significant principal components in the transformed space; LDA projects the original data into one dimension, the component that guarantees the highest separability between classes, where the helpfulness for classification is clear; Isomap embedding, where each data point (trial) is connected to its two closest neighbours. Input vector from classes RH and LH are denoted as classes 1/0, marked in blue/red circles.

$$
g\left(x_{i}\right)=w^{\top} x_{i}+b=0
$$

The values of $w$ and $b$ are not unique, and there are an infinity of hyperplanes that could divide classes RH and LH; however, to provide a good generalisation, SVM need to find the optimal hyperplane, the one that maximises the margin separation between the two classes. Defining classes $y_{i}=\{R H, L H\}$ as $y_{i}=\{1,-1\}$, the decision rule is defined by equation 8 , and the margin is defined as $\frac{2}{\|w\|}$. Equation 8 can be rewritten as $y_{i}\left(w^{\top} x_{i}+b\right) \geqslant$ $1, \forall_{i}$ and learning the SVM can be formulated as an optimisation problem (equation 9).

$$
\begin{aligned}
w^{\top} x_{i}+b \geqslant 1, & \text { if } \quad x_{i} \in R H \\
w^{\top} x_{i}+b \leqslant-1, & \text { if } \quad x_{i} \in L H
\end{aligned}
$$

$$
\text { Maximize } \frac{2}{\|w\|} \quad \text { subject to } \quad y_{i}\left(w^{\top} x_{i}+b\right) \geqslant 1, \forall_{i}
$$

\subsubsection{Decision Trees}

As the name implies, Decision Trees (DT) are supervised, tree-based models, where the input space (all existing BCI trials) is recursively divided into two or more subspaces based on the discriminative power of the existing features 42 . A decision tree starts with a root node, which is the feature whose split is more informative for class assignment (e.g. Hjorth activity parameter), and from the root node several branches arise, creating several other nodes, called internal or test nodes. Each node in the tree is a feature (e.g. power spectral density of alpha band in channel 1, adaptive regression model coefficients in channel 2) and represents a condition that must be tested. Branches are the possible solutions for the test conditions at each node, represented by each node's possible values (for discrete features, they are all the existing categories in the considered subspace; for continuous features, which is the case of this BCI task, each branch represents a certain range of values). The classification of a trial $\mathbf{x}$ is achieved by following its path along the nodes, from the root to a terminal node (leaf), according to the conditions tested along the way. Leaves are nodes with only incoming branches (they are not further divided), each one assigned to a class - typically, each leaf assumes the class with the highest fraction of patterns included in its subspace: for instance, a trial that ends in a leaf with 6/10 RH examples and 4/10 LH examples will be assigned to RH class.

\subsection{Sampling Strategies}

To estimate the performance of a classifier, it's necessary to determine its error rate across the entire population of examples, which is impractical in real-world applications (the whole population is not available to study). Sampling strategies allow the 
partition of the existing examples to train and test the classification models, and provide an estimate of the true error. Considering a motor imagery context, these approaches split a subject's trials into two groups of examples: training examples, where a percentage of trials is used to build the classification model and the test examples, where trials never seen by the model are used for evaluation. In this section, we review the most commonly used strategies in BCI scenarios $43 / 44 / 45 / 46$.

\subsubsection{Holdout method}

Holdout method randomly divides the dataset in $k=$ 2 sets, typically considering a $70 \%-30 \%$ or $80 \%$ $20 \%$ split for training/test $\frac{47}{4}$. The model is constructed using the training set while the performance metrics are computed using the test set. This method is rather simple, yet comprises some limitations: holdout may be subjected to "unlucky" splits, where the training set may not be representative of the total examples 36 .

\subsection{2. $K$-fold cross-validation}

In k-fold cross-validation, the dataset is partitioned in $k$ distinct folds, where generally $k$ is commonly set to 10 33. These folds will rotate in such a way that all of them are used for training and testing the model: $k-1$ folds are used as training set and the remaining fold (test set) for evaluation, and the procedure is performed $k$ times, rotating the position of the test set. The final error estimation is produced according to equation 10 , by averaging the individual errors $e_{i}$ of each fold. This work considers a 10fold cross-validation scheme. The trade-off between bias and variance of a classifier is influenced by the choice of $k$ : a small $k$ provides a larger bias but low variance, whereas a larger $k$ produces error estimates closer to the true error (low bias), although increasing the variance.

$$
\text { error }=\frac{1}{k} \sum_{i=1}^{k} e_{i}
$$

\subsubsection{Leave-One-Out}

When $k=N$ (with $N$ total examples), the sampling scheme is called Leave-One-Out (LOO), where the model is designed using $N-1$ examples and tested using the left-out example, and the process is repeated $N$ times. Thus, the final error estimate is computed by averaging all the $N$ individual errors. LOO uses only one example for testing, which produces a large variance in the error estimation; however, since all the remaining $N-1$ examples are used for the design of the classifier, the average test error estimate (over all $N$ runs), is an accurate estimate of the true error, resulting in a low bias.

\subsection{Performance Evaluation}

In $\mathrm{BCI}$ contexts, accuracy is the most commonly used metric to evaluate the performance of a classification approach 814 and represents the rate of correctly classified patterns. There are, however, several other important metrics to properly evaluate a given approach, and they are, as Accuracy, based on a confusion matrix (Table 2) 48. A confusion matrix describes the performance of a classifier on the basis of its predictions (Predicted Class), versus the true, known classes (Actual Class). Table 2 shows a confusion matrix for binary classification problem, such as the motor imagery task in this work, where "movement of right hand" (RH) is considered the positive class and "movement of left hand" (LH) the negative class. In what follows, we provide a brief explanation of the current performance measures used in MI tasks 4950 .

\begin{tabular}{lllc}
\multicolumn{4}{c}{ Table 2. Confusion Matrix } \\
\hline & \multicolumn{2}{c}{ Actual } \\
\cline { 2 - 4 } & & Negative & Positive \\
\hline \multirow{2}{*}{ Predicted } & $\begin{array}{c}\text { Negative } \\
\text { Positive }\end{array}$ & $\begin{array}{c}\text { True Negative (TN) } \\
\text { False Positive (FP) }\end{array}$ & $\begin{array}{c}\text { False Negative (FN) } \\
\text { True Positive (TP) }\end{array}$ \\
\hline
\end{tabular}

In the context of BCI motor imagery tasks, the entries in the above confusion matrix have the following meaning: True Positives (TP) and True Negatives $(\mathrm{TN})$ represent the number of trials correctly classified as RH and LH movement, respectively. False Positives (FP) are the number of LH trials that are misclassified as RH movement and False Negatives (FN) represent the number of RH trials 
misclassified as LH movement. For binary classification problems, a confusion matrix is the starting point for the definition of fundamental performance metrics: Accuracy, Sensitivity, Specificity, Precision and Negative Predictive Value. Herein we explain each one with a specific focus on motor imagery tasks.

Accuracy (Acc), as above mentioned, represents the proportion of correctly classified patterns: the proportion of $\mathrm{RH}$ and $\mathrm{LH}$ movement predictions that were correct (classified as RH and LH trials, respectively), and is calculated using equation 11 .

$$
\mathrm{Acc}=\frac{T P+T N}{T P+T N+F P+F N}
$$

Sensitivity (Sens) reports on the proportion of positive patterns correctly classified; that is; out of all the existing true $\mathrm{RH}$ trials, how many were correctly identified (equation 12). Specificity (Spec) is similar, but respects to the negative class: the proportion of LH trials correctly identified (equation 13.

$$
\begin{aligned}
& \text { Sens }=\frac{T P}{T P+F N} \\
& \text { Spec }=\frac{T N}{T N+F P}
\end{aligned}
$$

Precision (Prec) represents the proportion of the predicted positive patetrns that were correctly classified: out of all trials predicted as RH movement, the ones that were in fact representing RH movements (equation 14). The Negative Predictive Value or Negative Prediction (PrecNeg), shows how many predicted negative patterns were correctly identified: of all trials predicted as LH movement, how many were in fact LH movement trials (equation 15).

$$
\begin{gathered}
\text { Prec }=\frac{T P}{T P+F P} \\
\text { PrecNeg }=\frac{T N}{T N+F N}
\end{gathered}
$$

Precision, along with Sensitivity are the two required terms to compute F-measure (equation 16). F-measure balances the trade-off between Precision and Sensitivity, being defined as a weighted measure of both (an "harmonic mean"). In any context, including motor imagery tasks, achieving a high sensitivity and high precision is difficult, and an effort to improve the performance of one causes a performance decrease in the other. For instance, achieving a maximum Sensitivity would be straightforward: the classifier would simply set all labels to "RH". For maximum Precision, however, the classifier could not account for any false positives (LH trials classified as RH), which is incompatible with setting all trials to RH for maximum Sensitivity. Fmeasure then traduces the ability of the classifier to provide strong results in both, by using equation 16 .

$$
\text { F-measure }=\frac{2 * \operatorname{Prec} * \text { Sens }}{\operatorname{Prec}+\text { Sens }}
$$

Besides being used for feature selection, AUC values can also be used for evaluating the classification performance, as previously explained.

\section{Experiments and Discussion of Results}

Fig. 6 gives an overview of the experiments conducted in this work. The datasets with the extracted features (PSD, H, AR and CWT) from the BCI signals of BCI competitions II and III are analysed. In the first step, for each subject, six different datasets were constructed based on the results of FS and FT algorithms: original dataset, FS dataset, $\mathrm{PCA}_{\text {Kaiser }}$ dataset, $\mathrm{PCA}_{\text {Scree }}$ dataset, Isomap dataset and LDA dataset. After that, the datasets were tested with four different classifiers, each using three sampling schemes (note that LDA is not coupled with FLD). Therefore, 252 different combinations are evaluated. The accuracy, sensitivity, specificity, precision, negative precision, F-measure and AUC values were determined for each one of those 252 combinations.

Since this work comprises an extensive set of simulations, this section will be divided into three main subsections: "Feature Selection Results", "Feature Transformation Results" and "Classification Results", to ease the discussion and simplify the analysis by the reader. 


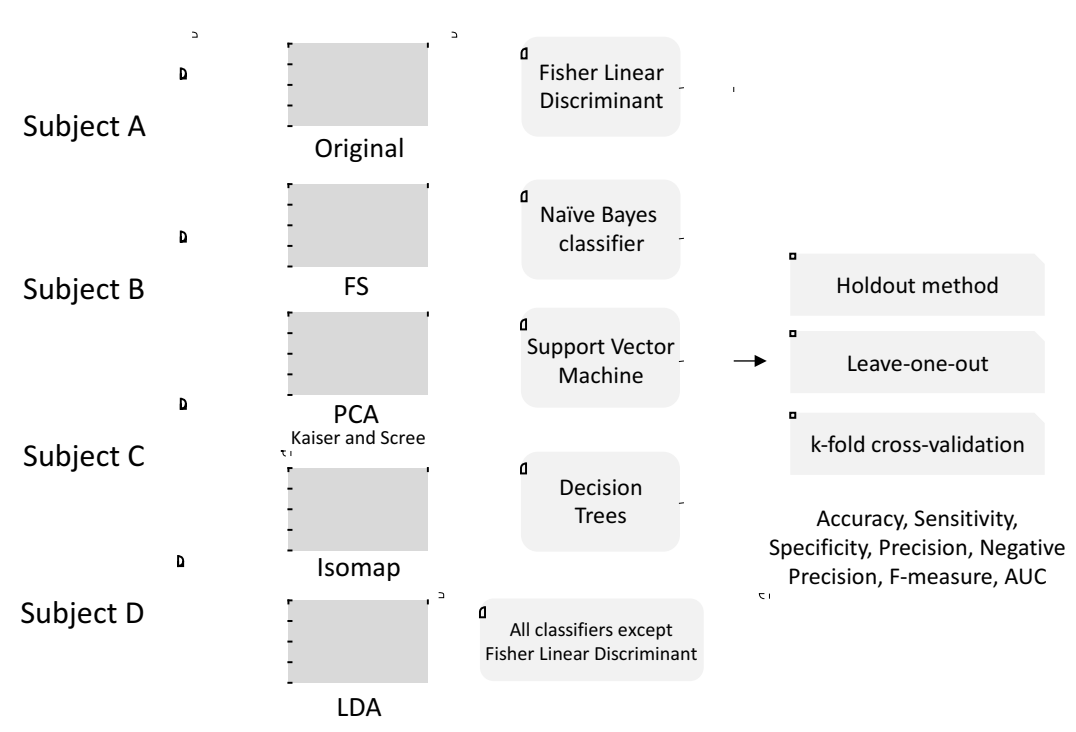

Fig. 6. Overview of the experiments.

\subsection{Feature Selection Results}

The feature selection results are summarised in Table 3, where each original dataset was analysed to select a subset of the most discriminative features. Considering all subjects, the features related to PSD in the beta frequency, AR and CWT coefficients were the most frequently selected as discriminative. On the contrary, the discriminative power of Hjorth parameters could not be verified for all the subjects. Furthermore, although it is not possible to have the same exact criteria for selection among all subjects, the AUC values, $r_{p b}$ and $\mathrm{Qui}^{2}$ produce similar results, which is important to move towards an approach that returns reliable results, independently of the test subject.

Table 3. Selected features and criteria used for each dataset.

\begin{tabular}{lcccc}
\hline & Subject 1 & Subject 2 & Subject 3 & Subject 4 \\
\hline AUC & $>0.6$ & $>0.57$ & $>0.57$ & $>0.57$ \\
F-score & $>0.2$ & $>0.05$ & $>0.1$ & $>0.15$ \\
IG & $>0.1$ & $>0.02$ & $>0.02$ & $>0.02$ \\
$\mathbf{r}_{\mathbf{p b}}$ & $>0.2$ & $>0.15$ & $>0.2$ & $>0.2$ \\
Qui $^{2}$ & $>10$ & $>10$ & $>10$ & $>10$ \\
Selected Features & {$[1,3],[5,9]$,} & {$[1,3],[6,8], 14$,} & $2,4,12$, & $19,[22,28]$ \\
& {$[18,20], 22,[26,28]$} & $16,19,20,28$ & 21,29 & \\
\hline
\end{tabular}

\subsection{Feature Transformation Results}

Feature Transformation results are shown in Table 4 and herein explained in what follows. LDA and
Kaiser criterion are performed automatically: LDA reduces the input data to one dimension and Kaiser criteria discards principal components with eigenvalues below 1. For the remaining criteria (scree test and isomap embedding), a more thorough analysis is required: the number of components for which the residual variance ceases to decrease significantly needs to be inspected for each subject. Concerning the PCA results, scree test always suggests a higher number of dimensions. In fact, although kaiser criterion keeps the components responsible for the great majority of data variance, there can still be components with low variance that can be helpful for discriminating class-membership. Therefore, scree criteria suggests keeping between 25 to 28 dimensions.

Table 4. Extracted dimensions for each dataset.

\begin{tabular}{lcccc}
\hline & Subject 1 & Subject 2 & Subject 3 & Subject 4 \\
\hline LDA & 1 & 1 & 1 & 1 \\
PCA $_{\text {Kaiser }}$ & 9 & 11 & 11 & 13 \\
PCA $_{\text {Scree }}$ & 25 & 28 & 26 & 25 \\
Isomap & 6 & 20 & 15 & 25 \\
\hline
\end{tabular}

\subsection{Classification Results}

The classification results are summarised in Table 5 for all subjects. Table 5 presents the results for FLD, NB, SVM and DT using holdout, k-fold crossvalidation and LOO: for each type of FS and FT 
(including also the original dataset), the best value achieved in each performance metric is shown, including the combination of classifier and sampling method that provided such value. In some cases, several classifiers and/or sampling methods are presented, meaning that they achieved the same results. Also, the best value considering all combinations is marked in bold. Overall, FLD and SVM classifiers showed a robust behaviour, being the top two classifiers achieving the highest performance results for the majority of considered combinations. Generally, FS and FT methods seem to improve the baseline classifications (with the original data), with the exception of Isomap embedding, which performs poorly, especially for subjects 3 and 4 . The comprehensive set of techniques used for FS achieves better results than PCA and Isomap, although not LDA. Furthermore, the datasets constructed using Scree test returned better results than the ones constructed using the Kaiser criterion, which indicates that for these subjects there are components with low variance that contribute for the discriminative process. Nevertheless, it is LDA that outperforms the remaining FT and FS methods, where an impressive reduction is performed (one dimension). These observations suggest that (i) the combination of the chosen FS methods may be a feasible approach for motor imagery scenarios, with higher performance than other FT methods, (ii) isomap embedding is responsible for the lowest performance, indicating that BCI data benefits the most from linear mappings, such as PCA and LDA, (iii) Scree test maintains several components with low variance that may be crucial for discriminations, and therefore should be preferred over Kaiser criterion, and finally (iv) linear and supervised FT approaches (LDA) are the most appropriate for BCI data, achieving good performance indicators and a considerable reduction of the problem's dimensionality, decreasing the computational cost of classification tasks.

It must be noted that some improvements in performance are associated with the holdout method; however, the comparison between datasets and classifiers using only this method might not be the most appropriate approach, since different random partitions of data could have different results ("lucky splits" or "unfortunate splits" may happen, as previously explained). For that reason, Table 6 focuses on the results for LOO for all classifiers: since FLD is one of the most extensively used machine learning algorithms in BCI research, the results obtained with FLD and LOO for the original datasets define the baseline classification for this analysis. The feature reduction using LDA, coupled either with NB or SVM, consecutively improved the baseline results for all subjects, which suggests that LDA is a suitable approach for dimensionality reduction in BCI data. DT performed poorly, and thus is not considered to be a valid approach to use on this type of problem. Another observation is that, when considering only the LOO results, SVM is still one of the top classifiers, although it shares its place in the podium with NB classifier, which is increasingly receiving a renewed attention for motor imagery tasks in recent years 338 .

Furthermore, we also present the k-fold crossvalidation accuracy results for the original and LDA reduced datasets, considering all classifiers, in order to assess the variance in the predictions of each classifier (only $\mathrm{k}$-fold provides an estimate on the variance of the predictions, through the standard deviation of the performance achieved in each fold). The k-fold results (Table 7), confirm that transforming the datasets with LDA improves the accuracy results, with also lower standard deviations than for the same approach in the original datasets. BCI datasets can therefore be extremely reduced with LDA without significant loss of information. Furthermore, NB and SVM achieved frequently similar results, although generally SVM results are slightly better.

\section{Conclusions and Future Work}

In this work, a comprehensive study of the stages that compose the machine learning block of BCI systems is performed. Existing approaches applied in several stages - feature selection, feature transformation, classification and performance evaluation are explained and discussed in detail, in order to address the following research question: "What are the steps in the classification process that we should 
Table 5. Overall performance results.

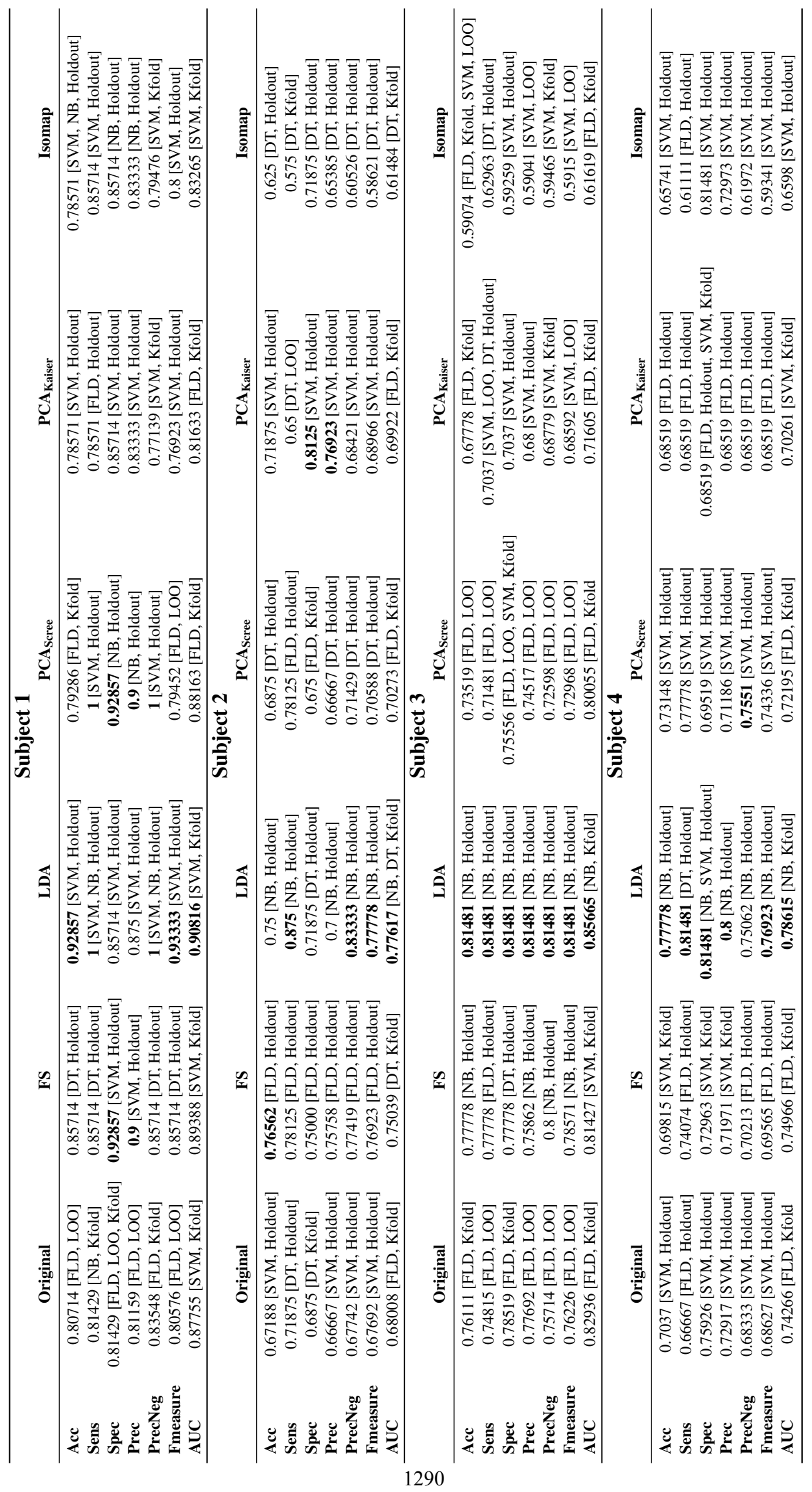


Table 6. Leave-one-out performance results: FDA baseline versus Best Approach.

\begin{tabular}{|c|c|c|c|c|c|c|c|c|}
\hline & \multicolumn{2}{|c|}{ Subject 1} & \multicolumn{2}{|c|}{ Subject 2} & \multicolumn{2}{|c|}{ Subject 3} & \multicolumn{2}{|c|}{ Subject 4} \\
\hline & FDA Baseline & Best Approach & FDA Baseline & Best Approach & FDA Baseline & Best Approach & FDA Baseline & Best Approach \\
\hline Acc & 0.80714 & 0.817143 [SVM, LDA] & 0.61562 & 0.6875 [NB, LDA] & 0.76667 & 0.77037 [NB, LDA] & 0.67963 & 0.70556 [SVM, LDA] \\
\hline Sens & 0.8 & $0.97143[\mathrm{SVM}, \mathrm{LDA}]$ & 0.63125 & 0.775 [NB, SVM, LDA] & 0.74815 & $0.7963[\mathrm{NB}, \mathrm{LDA}]$ & 0.66296 & $0.75926[\mathrm{NB}, \mathrm{LDA}]$ \\
\hline Spec & 0.81429 & 0.82857 [SVM, FS] & 0.6 & 0.675 [FLD, SVM, FS] & 0.78519 & 0.79259 [SVM, LDA] & 0.6963 & 0.71481 [SVM, FS] \\
\hline Prec & 0.81159 & 0.82278 [NB, LDA] & 0.61212 & 0.66452 [FLD, FS] & 0.77692 & 0.78125 [SVM, LDA] & 0.68582 & 0.69922 [SVM, FS] \\
\hline PrecNeg & 0.80282 & 0.96429 [SVM, LDA] & 0.61935 & 0.72727 [NB, LDA] & 0.75714 & $0.78516[\mathrm{NB}, \mathrm{LDA}]$ & 0.67384 & 0.72917 [NB, LDA] \\
\hline Fmeasure & 0.80576 & $0.88312[\mathrm{SVM}, \mathrm{LDA}]$ & 0.62154 & 0.71264 [NB, LDA] & 0.76226 & 0.77617 [NB, LDA] & 0.6742 & $0.7193[\mathrm{NB}, \mathrm{LDA}]$ \\
\hline AUC & 0.72648 & 0.73643 [NB, LDA] & 0.57532 & $0.61186[\mathrm{FLD}, \mathrm{FS}]$ & 0.69708 & $0.70065[\mathrm{NB}, \mathrm{LDA}]$ & 0.62717 & 0.63695 [SVM, FS] \\
\hline
\end{tabular}

Table 7. K-fold crossvalidation accuracy results: Original Data versus LDA Projection.

\begin{tabular}{|c|c|c|c|c|c|c|c|c|}
\hline & \multicolumn{2}{|c|}{ Subject 1} & \multicolumn{2}{|c|}{ Subject 2} & \multicolumn{2}{|c|}{ Subject 3} & \multicolumn{2}{|c|}{ Subject 4} \\
\hline & Original & LDA & Original & LDA & Original & LDA & Original & LDA \\
\hline FLD & $0.8 \pm 0.1338$ & n.a. & $0.6062 \pm 0.0782$ & n.a. & $0.7611 \pm 0.0575$ & n.a. & $0.6722 \pm 0.0912$ & n.a. \\
\hline NB & $0.7643 \pm 0.1169$ & $0.8643 \pm 0.0786$ & $0.6062 \pm 0.1044$ & $0.6906 \pm 0.1015$ & $0.6611 \pm 0.0479$ & $0.7685 \pm 0.0747$ & $0.6500 \pm 0.0332$ & $0.7037 \pm 0.0262$ \\
\hline SVM & $0.7714 \pm 0.0811$ & $0.8714 \pm 0.0563$ & $0.6156 \pm 0.1170$ & $0.6875 \pm 0.0859$ & $0.7537 \pm 0.0630$ & $0.7667 \pm 0.0518$ & $0.6648 \pm 0.0758$ & $0.7093 \pm 0.0454$ \\
\hline DT & $0.7714 \pm 0.1421$ & $0.8214 \pm 0.1078$ & $0.6438 \pm 0.0886$ & $0.6594 \pm 0.0772$ & $0.6185 \pm 0.0574$ & $0.7167 \pm 0.0531$ & $0.6000 \pm 0.0820$ & $0.6759 \pm 0.0587$ \\
\hline
\end{tabular}

worry about?". To that end, a thorough study of four motor imagery datasets was performed. Each dataset was analysed using five methods for feature selection (AUC, F-score, Information Gain, $r_{p} b$ and $\mathrm{Qui}^{2}$ ), three methods for feature transformation (LDA, PCA with Kaiser and Scree criteria and Isomap), four different classifiers (FLD, NB, SVM and DT) and three sampling strategies (holdout, kfold cross-validation and leave-one-out), resulting in 252 different combinations (LDA is not coupled with FLD). Regarding the dimensionality reduction of motor imagery dataset, all FS and FT approaches improved the baseline results. The set of FS techniques tested seems to be a feasible approach, where features related to the PSD in the beta bands, and two specific AR and CWT coefficients seem to be the most discriminative for motor imagery tasks. Also, FS seemed to be better for discrimination than some FT techniques, such as PCA and Isomap, although not LDA. In particular, Isomap performed poorly, and does not seem to be an appropriate reduction method for motor imagery. BCI data seems therefore to benefit the most from linear mappings, rather than nonlinear ones, and in particular from linear, supervised methods such as LDA, which has shown very good results. Regarding classifiers, none has strongly outperformed all others for all considered combinations and subjects, although SVM and NB showed a robust behaviour, particularly when coupled with LDA. Regarding the sampling schemes, although LOO produces a better estimate of the true performance error, it has a large variance. On the other hand, k-fold cross-validation, with a proper value of $k$, is also able to accurately approximate the true error, while providing a meaningful insight on the variance between estimates, which helps in establishing a measure of confidence in the designed classification model.

Facing these results, as a final conclusion of this work, it is clear that future research in this area should focus on transformation techniques for dimensionality reduction, in particular on the development/application of supervised linear approaches. However, a more thorough exploration of Naive Bayes classifiers for motor imagery problems, could also constitute an important contribution for knowledge. Performance results should be evaluated with $\mathrm{k}$-fold cross-validation in order to provide an estimate of how much the classifier varies in its predictions. As a final remark, the validation of these results could benefit from the analysis of different BCI datasets with more subjects and more EEG channels in order to manage higher groups of features in each trial.

\section{Acknowledgments}

The authors would like to dedicate this work to the memory of our colleague Professor Pedro J. GarcíaLaencina who sadly passed away during the course of this work. 


\section{References}

1. L. F. Nicolas-Alonso, J. Gomez-Gil, Brain computer interfaces, a review, Sensors 12 (2) (2012) 12111279.

2. S. N. Abdulkader, A. Atia, M. Mostafa, Brain computer interfacing: Applications and challenges, Egyptian Informatics Journal 16 (2) (2015) 213-230.

3. F. Lotte, M. Congedo, A. Lécuyer, F. Lamarche, B. Arnaldi, A review of classification algorithms for eeg-based brain-computer interfaces, Journal of neural engineering 4 (2) (2007) 1.

4. A. Bashashati, M. Fatourechi, R. K. Ward, G. E. Birch, A survey of signal processing algorithms in brain-computer interfaces based on electrical brain signals, Journal of Neural engineering 4 (2) (2007) 32.

5. S. Sun, J. Zhou, A review of adaptive feature extraction and classification methods for eeg-based braincomputer interfaces, in: Proc. IEEE International Joint Conference on Neural Networks (IJCNN), 2014, pp. 1746-1753.

6. R. Salazar-Varas, D. Gutiérrez, An optimized feature selection and classification method for using electroencephalographic coherence in brain-computer interfaces, Biomedical Signal Processing and Control 18 (2015) 11-18.

7. I. Koprinska, Feature selection for brain-computer interfaces, in: Pacific-Asia Conference on Knowledge Discovery and Data Mining, Springer, 2009, pp. 106-117.

8. G. Rodríguez-Bermúdez, P. J. García-Laencina, J. Roca-Dorda, Efficient automatic selection and combination of eeg features in least squares classifiers for motor imagery brain-computer interfaces, International journal of neural systems 23 (4) (2013) $1-17$.

9. S. Bhattacharyya, A. Sengupta, T. Chakraborti, A. Konar, D. Tibarewala, Automatic feature selection of motor imagery eeg signals using differential evolution and learning automata, Medical \& biological engineering \& computing 52 (2) (2014) 131-139.

10. S. Park, H. Hwang, J. Lim, J. Choi, H. Jung, C. Im, Evaluation of feature extraction methods for eegbased brain-computer interfaces in terms of robustness to slight changes in electrode locations, Medical \& biological engineering \& computing 51 (5) (2013) 571-579.

11. A. Schlogl, C. Neuper, G. Muller, B. Graimann, G. Pfurtscheller, Bci competition ii, http://www . bbci.de/competition/ii/(2002).

12. A. Schlogl, C. Neuper, G. Muller, B. Graimann, G. Pfurtscheller, Bci competition iii, http://www . bbci.de/competition/iii/(2005).

13. M. Billinger, C. Brunner, C. Neuper, Classification of adaptive autoregressive models at different sampling rates in a motor imagery-based bci, in: Fourth International BCI Meeting, Pacific Grove, CA, USA, 2010.

14. P. J. García-Laencina, G. Rodríguez-Bermudez, J. Roca-Dorda, Exploring dimensionality reduction of eeg features in motor imagery task classification, Expert Systems with Applications 41 (11) (2014) 5285-5295.

15. M. M. Suarez-Alvarez, D. T. Pham, M. Y. Prostov, Y. I. Prostov, Statistical approach to normalization of feature vectors and clustering of mixed datasets, in: Proc. R. Soc. A, The Royal Society, 2012.

16. M. Seoane Santos, P. Henriques Abreu, P. J. GarcíaLaencina, A. Simão, A. Carvalho, A new clusterbased oversampling method for improving survival prediction of hepatocellular carcinoma patients., Journal of Biomedical Informatics 58 (2015) 49-59.

17. P. Henriques Abreu, M. Seoane Santos, M. Henriques Abreu, B. Andrade, D. Castro Silva, Predicting breast cancer recurrence using machine learning techniques: A systematic review, ACM Computing Surveys 49 (3) (2016) 1-40.

18. I. Guyon, A. Elisseeff, An introduction to variable and feature selection, Journal of machine learning research 3 (2003) 1157-1182.

19. L. Van Der Maaten, E. Postma, J. Van den Herik, Dimensionality reduction: a comparative, J Mach Learn Res 10 (2009) 66-71.

20. M. Prasad, Online feature selection for classifying emphysema in hrct images, International Journal of Computational Intelligence Systems 1 (2008) 127133.

21. N. Spolaôr, H. D. Lee, W. S. R. Takaki, F. C. Wu, Feature selection for multi-label learning: A systematic literature review and some experimental evaluations, International Journal of Computational Intelligence Systems 8 (2) (2015) 3-15.

22. B. Blankertz, L. Acqualagna, S. Dähne, S. Haufe, M. Schultze-Kraft, I. Sturm, M. Ušćumlic, M. A. Wenzel, G. Curio, K.-R. Müller, The berlin braincomputer interface: Progress beyond communication and control, Frontiers in Neuroscience 10 (2016) 124.

23. L. B. Felix, A. d'Affonsêca Netto, F. d. S. Ranaudo, A. M. F. L. M. Sá, et al., A spatial coherence-based vision-free brain-computer interface using auditory selective attention, MedicalExpress 2 (6) (2015) 1-6.

24. K. R. Müller, M. Krauledat, G. Dornhege, G. Curio, B. Blankertz, Machine learning techniques for braincomputer interfaces, Biomed Tech 49 (1) (2004) 1122.

25. V. T. Bai, et al., Feature selection in brain computer 
interface using genetics method, in: Proc. IEEE International Conference on Computer and Information Technology, 2015, pp. 270-275.

26. M. S. Treder, A. Bahramisharif, N. M. Schmidt, M. AJ van Gerven, B. Blankertz, Brain-computer interfacing using modulations of alpha activity induced by covert shifts of attention, J Neuroeng Rehabil 8 (24) (2011) 1-9.

27. J. A. Hanley, B. J. McNeil, The meaning and use of the area under a receiver operating characteristic (roc) curve., Radiology 143 (1) (1982) 29-36.

28. T. K. Ho, M. Basu, Complexity measures of supervised classification problems, IEEE transactions on pattern analysis and machine intelligence 24 (3) (2002) 289-300

29. T. M. Mitchell, Machine Learning, 1st Edition, McGraw-Hill, 1997.

30. R. F. Tate, Correlation between a discrete and a continuous variable. point-biserial correlation, The Annals of mathematical statistics 25 (3) (1954) 603607.

31. P. E. McKight, J. Najab, Kruskal-wallis test, Corsini Encyclopedia of Psychology (2010) 764-765.

32. E. Krivov, M. Belyaev, Dimensionality reduction with isomap algorithm for eeg covariance matrices, in: 2016 4th International Winter Conference on Brain-Computer Interface (BCI), 2016, pp. 1-4.

33. J. Marques de Sá, Pattern recognition: concepts, methods and applications, Springer Science \& Business Media, 2012.

34. J. C. Costa, P. J. G. Silva, R. M. Almeida, A. F. Infantosi, Validation in principal components analysis applied to eeg data, Computational and mathematical methods in medicine 2014.

35. A. Vallabhaneni, T. Wang, B. He, Brain computer interface, in: Neural engineering, Springer, 2005, pp. 85-121.

36. C. M. Bishop, Pattern Recognition and Machine Learning, Springer, 2006.

37. J. B. Tenenbaum, V. De Silva, J. C. Langford, A global geometric framework for nonlinear dimensionality reduction, science 290 (2000) 2319-2323.

38. S. Bhaduri, A. Khasnobish, R. Bose, D. Tibarewala, Classification of lower limb motor imagery using $\mathrm{k}$ nearest neighbor and naïve-bayesian classifier, in: Proc. IEEE International Conference on Recent Advances in Information Technology (RAIT), 2016, pp.
499-504.

39. M. Higger, F. Quivira, M. Akcakaya, M. Moghadamfalahi, H. Nezamfar, M. Cetin, D. Erdogmus, Recursive bayesian coding for bcis, IEEE Transactions on Neural Systems and Rehabilitation Engineering (99) (2016) 1.

40. S. P. Luttrell, Partitioned mixture distribution: An adaptive bayesian network for low-level image processing, IEE Proc Vision, Image Signal Process 141 (4) (1994) 251-260.

41. B. Boser, I. Guyon, V. Vapnik, A training algorithm for optimal margin classifiers, in: In Proceedings of the annual workshop on Computational learning theory, 1992, pp. 144-152.

42. J. R. Quinlan, Simplifying decision trees, International journal of man-machine studies 27 (3) (1987) 221-234.

43. M. Billinger, I. Daly, V. Kaiser, J. Jin, B. Z. Allison, G. R. Müller-Putz, C. Brunner, Is it significant? guidelines for reporting bci performance, in: Towards Practical Brain-Computer Interfaces, Springer, 2012, pp. 333-354.

44. O. AlZoubi, I. Koprinska, R. A. Calvo, Classification of brain-computer interface data, in: Proceedings of the 7th Australasian Data Mining ConferenceVolume 87, Australian Computer Society, Inc., 2008, pp. 123-131.

45. M. T. F. Talukdar, S. K. Sakib, N. S. Pathan, S. A. Fattah, Motor imagery eeg signal classification scheme based on autoregressive reflection coefficients, in: Proc. IEEE International Conference on Informatics, Electronics \& Vision (ICIEV), 2014, pp. $1-4$.

46. P. S. Hammon, V. R. de Sá, Preprocessing and metaclassification for brain-computer interfaces, IEEE Transactions on Biomedical Engineering 54 (3) (2007) 518-525.

47. R. O. Duda, P. E. Hart, D. G. Stork, Pattern classification, 2nd Edition, John Wiley \& Sons, 2012.

48. R. Kohavi, F. Provost, Confusion matrix, Machine learning 30 (2-3) (1998) 271-274.

49. M. Dyson, An analysis of performance evaluation for motor-imagery based bci, J Neural Eng. 10 (3) (2013) $1-6$.

50. M. Clerc, L. Bougrain, F. Lotte, Brain-Computer Interfaces 1: Methods and Perspectives, John Wiley \& Sons, 2016. 